\title{
SUBTHRESHOLD IONIZATION OF WEAKLY BOUND COMPLEXES: STOCHASTIC ANALYSIS OF THE ROLE OF THE RYDBERG QUASICONTINUUM
}

\author{
V. M. AKULIN, $\uparrow$ V. AQUILANTI, B. BRUNETTI \\ and F. VECCHIOCATTIVI
}

Dipartimento di Chimica dell'Universitá, 06100 Perugia, Italy

\begin{abstract}
Recent evidence for subthreshold ionization (i.e. electron loss at energies less than anticipated from vertical transitions assuming adiabatic separation of nuclear motion) points at the role of nonadiabatic coupling of high Rydberg terms of molecules. Sinai's billiard model for the chaotic motion of the Rydberg electron, that leads to a diffusion over the energy ladder as a result of electronic-vibrational exchange, is suggested as the classical mechanism of autoionization. A quantum expression for the branching ratio between autoionization and spontaneous fluorescence is obtained and discussed with reference to experimental results on associative ionization in atomic collisions and on laser ionization of van der Waals diatomics.
\end{abstract}

KEY WORDS: Subthreshold, ionization, stochastic.

\section{INTRODUCTION}

The ionization of diatomic molecules and weakly bound diatomic complexes lead to molecular ions whose properties range between the following limiting cases: (a) ions where the two atoms are bound by chemical forces; (b) ions bound by weak interatomic forces, mainly ion-induced dipole interactions. Examples of molecules leading to ions of the first type are $\mathrm{NO}, \mathrm{O}_{2}, \ldots$ etc., while the heteronuclear van der Waals complexes $\mathrm{NeAr}, \mathrm{NEKr}, \mathrm{ArHg}, \mathrm{KrHg}, \ldots$. , lead to ions of the second type. The homonuclear $\mathrm{Ar}_{2}, \mathrm{Kr}_{2}, \mathrm{Hg}_{2}, \ldots$, are intermediate cases, because symmetry adds strength to bonds through exchange. It has to be noted that an important condition to produce ions of the second type is that the van der Waals molecule to be ionized is formed by atoms with different ionization potentials: higher is this difference, lower is the effect of the configuration interaction due to the charge exchange in the final ion.

In all these cases the ionization continuum is reached crossing the quasicontinuum of the molecular Rydberg states. For the first type of molecules the ionic core of the Rydberg states is characterized by a charge distribution totally or partially delocalized around the two nuclei, while for the second type the charge is predominantly

$\dagger$ Permanent address: General Physics Institute, Academy of Sciences, Moscow, USSR. 
localized on one of the two atomic partners, namely the one with the lower ionization potential.

There are increasing evidences that these diatomic molecules show different behaviour in the near-threshold ionization. In particular photoionization experiments carried out with molecules of the intermediate type, such as $\mathrm{Ar}_{2}, \mathrm{Kr}_{2}{ }^{1,2}$ or $\mathrm{Hg}_{2}{ }^{, 3}$ and of the second type, such as $\mathrm{NeAr}, \mathrm{NeKr}^{1,2}$ or $\mathrm{ArHg},{ }^{5} \mathrm{KrHg},{ }^{6}$ indicate that the ionization starts to occur under the vertical threshold. In particular, a close look to the experimental photoionization spectra seems to indicate a stronger effect with the second type heteronuclear van der Waals molecules. Less or no evidence so far has been found for this phenomenon with molecules leading to ions of the first type.

Analogies can be found in collision experiments between electronically excited atoms and other species where associative ionization occurs, $\mathrm{A}^{*}+\mathrm{B} \rightarrow \mathrm{AB}^{+}+\mathrm{e}^{-}$: it has been observed that the threshold is close to the vertical one for systems leading to ions characterized by chemical bond, such as $\mathrm{ArH}^{+7}$ or $\mathrm{NO}^{+}, 8,9$ while for heteronuclear systems, such as for example $\mathrm{Kr}^{*}+\mathrm{Hg} \rightarrow \mathrm{KrHg}^{+}+\mathrm{e}^{10}$ the apparent threshold for ionization is lower than the vertical one, evaluated using the standard approach of Born-Oppenheimer separation of the nuclear motion from the electron motion.

It has to be noted that the latter system $\left(\mathrm{KrHg}^{+}\right.$formation) appears to be particularly interesting because it has been studied by observing both photoionization $^{6}$ and collision associative ionization ${ }^{10}$ (see Figure 1). In this work a theoretical model, exemplified to the case of $\mathrm{KrHg}^{+}$formation, is proposed, which explains the

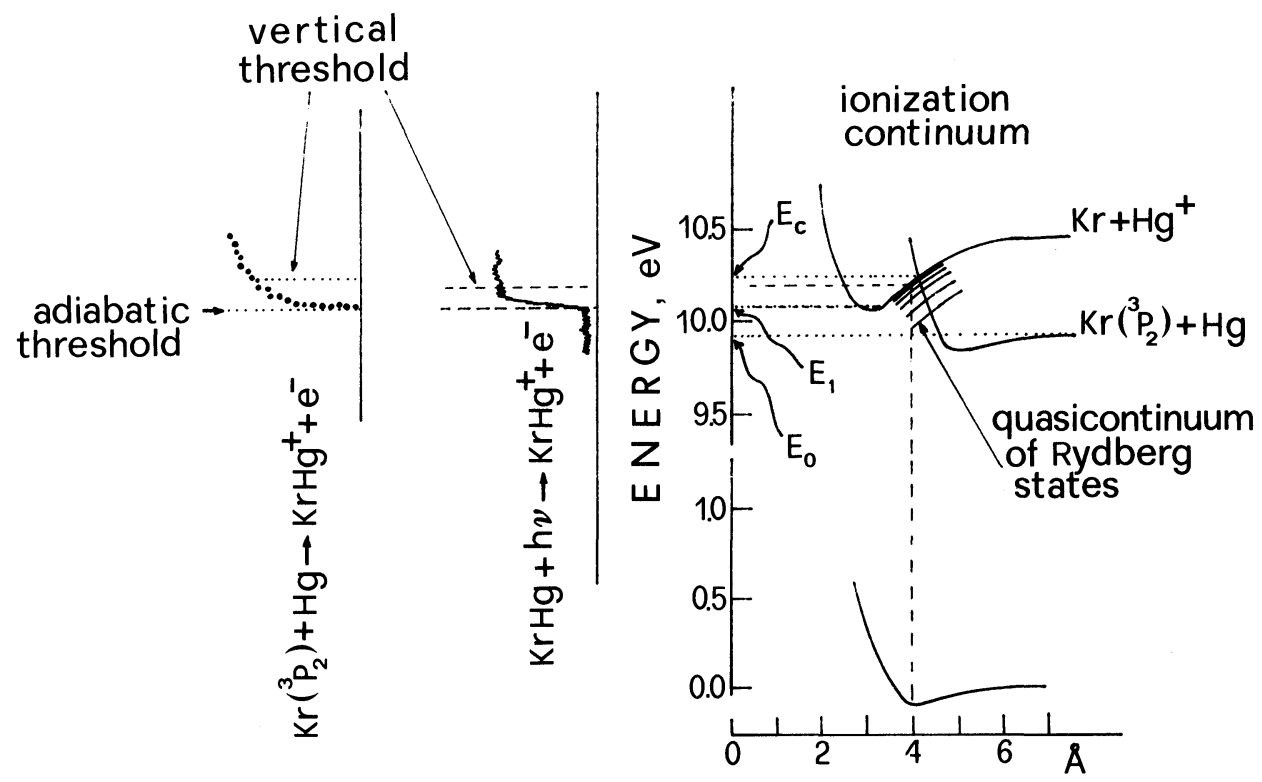

Figure 1 Sketch of potential energy curves of excited, Rydberg and ionized states of the mercurykrypton systems, for which both associative collisional ionization (Ref. 9) and photoionization cross sections (Ref. 5) are also shown. 
behaviour of the near-threshold ionization of heteronuclear van der Waals molecules as due to the coupling between electronic and nuclear motion in the Rydberg state dynamics. Miller and Morgner, ${ }^{11}$ in a unified treatment of Penning ionization and excitation transfer, have shown that the Rydberg states play an important role in the energy transfer processes in $\mathrm{Kr}^{*}+\mathrm{Hg}$ collisions: here it is suggested that the Rydberg states play an important role also in the near threshold ionization. In fact, for high Rydberg terms the typical period of electron motion may be of the order and even less than that of molecular vibrations. Therefore the adiabatic separation of nuclear and electronic degrees of freedom, valid for lower terms, now becomes invalid and the nonseparability of these two types of motion may lead to energy exchange between them. This implies that nonvertical, non Franck-Condon transitions begin to play a significant role (or in other words a series of nonadiabatic transitions follows the first, vertical, one) and must be taken into account.

Indeed, in the presence of an efficient energy exchange between electron and nuclear motion the sum of both contributions provide the energy for ionization, contrary to the Franck-Condon situation where all energy is supposed to be supplied to the electron only. We define this phenomenon as subthreshold ionization. It will become clear that the treatment explains why the phenomenon is increasingly important going from limiting cases (a) to (b) through the intermediate case.

The theoretical description of this phenomenon, which, due to the large atomic masses involved, cannot be attributed to pure quantum mechanical tunneling of nuclei, requires the explicit formulation of nonadiabatic coupling between nuclear motion and highly excited Rydberg states, which is not included in current theories: ${ }^{12}$ this difficult problem is tackled here by first showing that Rydberg molecular states provide an example of a quasicontinuum, thus providing the basis for a statistical approach to the dynamics.

\section{CLASSICAL ANALYSIS}

From classical mechanics we know that if the motion of even one particle in a three-dimensional space is nonseparable it becomes chaotic. ${ }^{13}$ In noncentral fields the motion of an electron is usually nonseparable (exceptions are systems showing some symmetry, the extreme case being the $\mathrm{H}_{2}^{+}$molecule). This implies that in molecules with an explicit asymmetry of the electron cloud the motion of the external electron should be chaotic when treated classically. Polar and van der Waals molecules likely possess such an asymmetry, and thus a highly excited electron of such a compound should behave stochastically. From this viewpoint, observation of photoionization at energy lower than that of vertical threshold for some heteronuclear van der Waals dimers, appears to provide further evidence for this phenomenon.

For the present discussion we take the system $\mathrm{Kr}^{*}-\mathrm{Hg}$ as an example (Figure 1). The system consists of two atoms approaching each other while one of them, $\mathrm{Kr}$, is at an excited electronic state. As a result of the interaction the excited $\mathrm{Kr}^{*}$ atom can capture an electron of the $\mathrm{Hg}$ atom filling thus a vacancy at its inner shells and 
pushing its excited electron to a highly excited Rydberg state. The system $\mathrm{KrHg}^{+}+$e now can be treated as a molecular ion in highly excited vibrational state, which is surrounded by the Rydberg electron moving on a stretched Kepler trajectory around the new charge center $\left(\mathrm{Hg}^{+}\right)$.

The Coulomb character of the interaction will manifest itself in the fact that the Rydberg electron after one turn around the $\mathrm{Hg}^{+}$atom tends to come back to the point it has started its motion. But the presence of the neutral $\mathrm{Kr}$ atom on its way prevents it to happen. As a result of scattering at this atom the electron changes its orbit and starts a new turn of Kepler's motion. The series of the same events leads to a situation analogous to Sinai's billiards ${ }^{14}$ and the orbital motion of the electron becomes chaotic as a result. ${ }^{15}$ It randomly changes orbits after each scattering event.

If now we take into account the vibrational motion of the molecular ion, the process of diffusion over energy becomes allowed. The energy of the electron thus becomes non-conserving. In other words, the vibrational energy of the molecular ion now can be transferred to the motion of the Rydberg electron. The large difference in velocities of the electron and nuclei allows to give the following evident classical description of the process. The electron being reflected by the moving $\mathrm{Kr}$ atom acquires an additional momentum of the order of $m_{e} V$, where $m_{e}$ is the mass of electron and $V$ is a typical velocity of the atom. Correspondent changing of the energy is of order $v_{e} V m_{e}$ where $v_{e}$ is the velocity of electron near the ion. The sign of the energy change may be both positive and negative with equal probability because of the random character of the electron motion. Diffusion of the electron over energy attains as a result.

\section{A QUANTUM FORMULA}

The classical analysis provides a background for a quantum stochastic treatment. The energy exchange between electron and ion is accompanied by a bremsstrahlung that occurs when the electron moves with high acceleration in the vicinity of the ion. It manifests itself in the spontaneous emission of a short-wave quantum of radiation and prevents further autoionization. Indeed, the molecule that has emitted a spontaneous quantum loses its energy and thus becomes excluded from the process of diffusion toward autoionization. Balance equation describing this process ${ }^{16}$ has the form

$$
\frac{d n_{k}}{d K}=M^{2}\left\{k\left(n_{k+1}-n_{k}\right)-(k-1)\left(n_{k}-n_{k-1}\right)\right\}-\gamma n_{k}
$$

where $n_{k}$ is occupation number of the $k$ th vibrational level; $K$ is the number of collision events; $\gamma$ is the probability of spontaneous emission during one collision; $M=\left(\hbar^{-1} m_{e} v_{e} X_{0} \sqrt{ } k\right)^{-2}$ is the Massey parameter; ${ }^{17} X_{0}$, zero-point vibration amplitude.

The solution of this equation allows to obtain the probability of the autoionization in terms of Bessel functions $K_{p}$. In the general case of a polyatomic molecular ion 
with $d$ vibrational degrees of freedom we obtain

$$
w_{i o n}=\frac{K_{d-1}\left(\sqrt{\frac{4 \gamma k_{0}}{M^{2}}}\right)}{K_{d-1}\left(\sqrt{\frac{4 \gamma k_{1}}{M^{2}}}\right)+\sqrt{\frac{\gamma}{M^{2} k_{1}}} K_{d}\left(\sqrt{\frac{4 \gamma k_{1}}{M^{2}}}\right)}
$$

where $k_{0}$ and $k_{1}$ are the number of vibrational quanta at the beginning and at the end of the process respectively.

\section{DISCUSSION}

This formula gives a universal dependence in terms of parameters which must be estimated for individual systems. In the spirit of this paper, only qualitative aspects will be considered and a detailed assessment of the theory towards experimental results requires further study.

We can estimate the probability $\gamma$ of spontaneous quenching after one kick multiplying the Einstein constant $A$ by the period of rotation of the electron round the center:

$$
\gamma \simeq \frac{4 \mu^{2} \omega^{3}}{3 \hbar c^{3}} \times \frac{2 \pi}{\omega} \simeq \frac{\alpha^{2}}{6 \pi^{3}}=2.0910^{-9}
$$

where $\alpha$ is the fine structure constant and $\mu$ is the dipole moment of the transition. Here we have taken into account that $\mu^{2} \omega^{3} \sim e^{2} v_{e}^{2}$ and $v_{e} / c \sim e^{2} / \hbar c=\alpha$. In fact $\gamma$ is the reciprocal of the quality factor for a typical atomic spectral line, i.e. the number of periods the system oscillates before quenching. For $\mathrm{KrHg}^{+}$the Massey parameter $M^{2} \sim 0.5$ and $k_{0}-k_{1} \sim 30$. Thus the parameter $\gamma k_{0} / M^{2} \simeq 1.210^{-7}$.

The variables $k_{1}$ and $k_{0}$ should be transformed to the energy of an incident particle when the process of associative ionization is considered. Parameters of such a transformation $E_{0}, E_{1}, E_{c}$ that characterize the molecular terms are introduced in Figure 1 (starting from $E_{0}=0$, estimates for $E_{1}$ and $E_{c}$ are 0.15 and $0.28 \mathrm{eV}$ respectively):

$$
\begin{gathered}
k_{1}\left(E_{\mathrm{rad}}\right)=\frac{E_{\mathrm{rad}}-E_{1}}{\hbar \omega_{\mathrm{vib}}} \\
k_{0}\left(E_{\mathrm{rad}}\right)=\frac{E_{1}-E_{0}}{\hbar \omega_{\mathrm{vib}}}-\frac{2 E_{1}-E_{c}-E_{0}}{\hbar \omega_{\mathrm{vib}}} \frac{E_{\mathrm{rad}}-E_{1}}{E_{c}-E_{1}}
\end{gathered}
$$

where $E_{\text {rad }}$ is the part of translational energy corresponding to a radial motion. Usually in the experiments the total translational energy $E$ is the variable parameter of a collision. So we have to average the ionization probability over different radial energies corresponding to the same total translational energy in order to obtain the 
values that could be compared with the experimental ones. For the one dimensional case

$$
w(E)=\int_{0}^{E} w_{\text {ion }}\left(k_{0}\left(E_{\mathrm{rad}}\right) ; k_{1}\left(E_{\mathrm{rad}}\right)\right) \frac{d E_{\mathrm{rad}}}{E}
$$

Or in other words, the total energy with an equal probability distributes between the rotational and the vibrational degree of freedom. In the multidimensional case almost all energy is transferred to vibrations.

In principle, rotations can play a role in the energy diffusion process. If necessary we can take them into account by the number of effective degrees of freedom $d$ and substitute the total translational energy of the particle in (2) using (4) and (5).

Results of calculations of $w_{\text {ion }}$ for the estimated parameters of the $\mathrm{KrHg}^{+}$system, are reported in Figure 2. The transformation (6) from the radial energy $E_{\mathrm{rad}}$ to the experimental collision energy $E$ will lead to an overall decrease of the effective ionization probability. One can see that for dimensionalities $d>1$ these dependencies resemble power laws. In fact this is the case, because at small $\gamma$ the

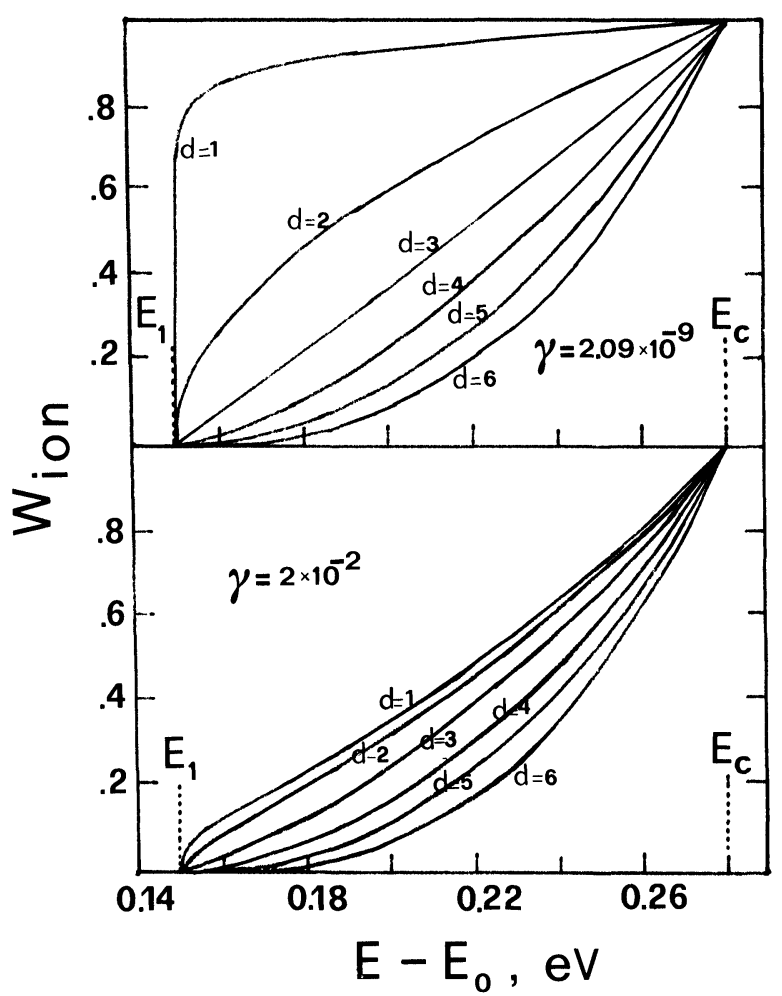

Figure 2 Subthreshold ionization probability as a function of radial energy for $\mathrm{Kr}^{*}-\mathrm{Hg}$, at different effective molecular degrees of freedom $d$ and for two values of the quenching probability $\gamma$. 
asymptotic formula which gives the power dependency

$$
K_{d}(\sqrt{z}) \sim z^{-d / 2}
$$

can be exploited. Then the parameter $\gamma$ does not enter any more in the expression for $w_{\text {ion }}$ and the latter takes a universal form

$$
w_{\text {ion }}=\left(\frac{k_{1}}{k_{0}}\right)^{(d-1) / 2}
$$

A slight logarithmic dependence on $\gamma$ persists only for the one dimensional case $d=1$.

A further viewpoint for the discussion is that $1-w_{\text {ion }}$ represents a probability of spontaneous emission. At small $\gamma$ this probability also exhibits the universal dependence on energy of nuclei, and this dependence is affected mainly by the number of degrees of freedom involved in the nuclear motion. We should keep in mind that the universal character of these dependencies is a consequence of the weakness of electron-photon interaction, characterized by the fine structure constant $\alpha=1 / 137$.

The process of autoionization may have quite different character when the decay of Rydberg states results from the irreversible structural change of the ion (intramolecular conversion) rather than from spontaneous emission. Among other possible processes of this kind we mention the backward transition from high Rydberg states of the molecular compound to the initial electron term corresponding to free motion of the colliding particles. This possibility always exists. These processes are of tunnel type and also take place only when the Rydberg electron hits the ion. Thereby the probability of the conversion as a result of a single kick is small. Nevertheless, it can be considerably larger comparing to that for bremsstrahlung. An illustration of how this effect modifies the ionization probability $w_{\text {ion }}$ is provided in Figure 2, where this probability calculated using formula (2) with $\gamma=210^{-2}$ is plotted as function of nuclear energy for different numbers of degrees of freedom. A recent paper ${ }^{18}$ can be consulted for more details and proofs of the theory outlined in this work.

\section{Acknowledgements}

Grants from ENEA, CNR and the EEC Science Program are gratefully acknowledged.

\section{References}

1. C. Y. Ng, Adv. Chem. Phys. 52, 263 (1982).

2. (a) P. M. Dehmer and E. D. Poliakoff, Chem. Phys. Lett. 73, 326 (1981); (b) P. M. Dehmer, J. Chem. Phys. 76, 1263 (1982).

3. S. H. Linn, C. L. Liao, J. M. Brom Jr and C. Y. Ng, Chem. Phys. Lett. 105, 645 (1984).

4. S. T. Pratt and P. D. Dehmer, J. Chem. Phys. 76, 3433 (1982).

5. S. H. Linn, J. M. Brom Jr., W. B. Tzeng and C. Y. Ng, J. Chem. Phys. 82, 648 (1985).

6. C. L. Liao and C. Y. Ng, 84, 1142 (1986).

7. (a) A. Niehaus, in "Physics of ionized gas", eds. B. Navinsek and J. Stefan, Ljubljana Institute (1976); (b) H. Morgner, Ph.D. Thesis, University of Freiburg (1976).

8. G. Ringer and W. R. Gentry, J. Chem. Phys. 71, 1902 (1979).

9. S. E. Nielsen and S. Dahler, J. Chem. Phys. 71, 1910 (1979). 
10. L. Appolloni, B. Brunetti, J. Hermanussen, F. Vecchiocattivi and G. G. Volpi, J. Chem. Phys. 87, 3804 (1987).

11. W. H. Miller and H. Morgner, J. Chem. Phys. 67, 4923 (1977).

12. (a) H. Nakamura, J. Phys. Soc. Japan 31, 574 (1971); (b) F. Koike and H. Nakamura, J. Phys. Soc. Japan 33, 1426 (1972); (c) W. H. Miller, J. Chem. Phys. 52, 3563 (1970).

13. A. J. Lichtenberg and M. A. Libermann, "Regular and stochastic motion", Springer, BerlinHeidelberg-New York-Tokyo (1983).

14. Ya. Sinai, Dokl. Aka. Nauk. 153, 1261 (1963) [Sov. Math. Doklady 4, 1818 (1963)].

15. (a) D. L. Shepelyanski, in "Chaotic behaviour in quantum systems", ed. G. Casati, Plenum Press (1985) p. 187; (b) G. Casati, B. V. Chirikov and D. L. Shepelyanski, Phys. Rev. A 53, 2525 (1984).

16. V. M. Akulin and N. V. Karlov, "Intensivnye resonansnye vzaimodeistviya" (Intense resonant interactions), Nauka, Moscow (1987) (to be published in English).

17. (a) H. S. W. Massey, Rept. Prog. Phys. 12, 248 (1949); (b) H. S. W. Massey, E. H. S. Burhop, "Electronic and ionic impact phenomena", Chapters VII and VIII, Oxford University Press, Oxford (1952).

18. V. Akulin, V. Aquilanti, B. Brunetti, F. Vecchiocattivi "Stochastic autoionization from the molecular Rydberg quasicontinuum", in "Quantum Chaos", eds. H. A. Cerderia, R. Ramaswamy, M. C. Gutzwiller and G. Casati, World Scientific, Singapore (1991), p. 423. 